Tohoku J. Exp. Med., 2009, 218, 25-33

\title{
Endothelial Cell Apoptosis is Responsible for the Formation of Coronary Thrombotic Atherosclerotic Plaques
}

\author{
Feng Xu, ${ }^{1,2, *}$ Yaning Sun, ${ }^{3,}$ Yuguo Chen, ${ }^{1,2}$ Yi Sun, ${ }^{1,2}$ Ruijian Li, ${ }^{1,2}$ Chunxi Liu, \\ Cheng Zhang, ${ }^{1,4}$ Rong Wang ${ }^{1}$ and Yun Zhang ${ }^{1,4}$ \\ ${ }^{1}$ Key Laboratory of Cardiovascular Remodeling and Function Research, Chinese Ministry of Education and \\ Chinese Ministry of Public Health, Jinan, China \\ ${ }^{2}$ Department of Emergency Medicine and Center of Chest Pain, Qilu Hospital of Shandong University, Jinan, \\ China \\ ${ }^{3}$ Department of Cardiology, Shandong Province Hospital affiliated to Shandong University, Jinan, China \\ ${ }^{4}$ Department of Cardiology, Qilu Hospital of Shandong University, Jinan, China
}

Ischemic manifestations of atherosclerosis are mainly due to thrombus formation upon superficially eroded (denudation of luminal endothelium) plaques or deeply ruptured (fibrous cap rupture) plaques. Eroded plaques, atherosclerotic plaques without rupture, are found common in young patients of sudden death with coronary thrombosis. Our study aimed to investigate the role of endothelial cell (EC) apoptosis in eroded plaque with thrombosis using an atherosclerotic rabbit model. Atherosclerotic plaques were established by post-balloon-injury high-cholesterol feeding in 33 rabbits. After three-month feeding, a 2-cm segment of plaque-rich abdominal aorta for each animal was clamped in vivo and filled with staurosporine, which induces endothelial apoptosis, or saline for 20 minutes. Three days later, serum lipids and high sensitive C-reactive protein (hs-CRP), a valuable inflammatory parameter, were quantified, and abdominal aorta angiography was conducted. In addition, immunohistochemistry staining was performed for all processed aortae. In the staurosporine-treated aorta, endothelium integrity of plaques was disrupted partially or in large areas, but fibrous cap rupture was not observed, the findings of which were similar to eroded plaques detected in human subjects. As compared to saline controls, staurosporine-treated rabbits showed higher apoptosis scores and thrombotic scores, and more angiographic overt thrombosis and histological thrombosis $(P<0.01$, respectively), despite the similar serum levels of lipids and hs-CRP. We further confirmed that apoptosis score was linearly associated with thrombotic score. These results suggest that endothelial apoptosis may be an independent risk factor for thrombosis. In conclusion, the increase in endothelial apoptosis is involved in the formation of thrombotic eroded plaques. - Atherosclerosis; Eroded plaque; Endothelial cell; Apoptosis; Sudden coronary death.

Tohoku J. Exp. Med., 2009, 218 (1), 25-33. (c) 2009 Tohoku University Medical Press

Ruptured and eroded atherosclerotic plaques with thrombosis are critical pathological processes of coronary atherosclerotic disease, which is responsible for ischemic sudden death and acute coronary syndrome (ACS) (Virmani et al. 2000; Naghavi et al. 2003a; Naghavi et al. 2003b). Although eroded plaque is less common than ruptured plaque, eroded plaque with thrombosis is still responsible for $30 \%-44 \%$ of acute coronary thrombotic events and is more common in young sudden death or female patients (van der Wal et al. 1994; Farb et al. 1996; Rosenson and Lowe 1998; Virmani et al. 2001; Kolodgie et al. 2004). It was reported that about $70 \%$ of thrombotic eroded plaque occurred in patients $<50$ years of age and the incidence in women was twice that in men. Eroded plaque appears to be very common in cases of sudden coronary death, however, diagnosis of eroded plaques before the occurrence of acute events is difficult mainly due to technological limitations. With only few clinical studies in this area, mechanisms of eroded plaque with thrombosis are also poorly understood (Virmani et al. 2000; Hayashi et al. 2005).

Endothelial denudation is a main characteristic of eroded plaque. Comparing to ruptured plaque, eroded plaque is not characterized by dissection, cleft in the fibrous cap, or lipid overflow under the thrombi. Only significant endothelial denudation at the plaque/thrombus interface was observed (Virmani et al. 2000). A pathological study showed that there was greater accumulation of extracellular matrix proteins, such as versican, hyaluronan and CD44, at

Received January 21, 2009; revision accepted for publication March 19, 2009.

"Equal contribution to this work.

Correspondence: Yuguo Chen, M.D., Ph.D., 107 Wenhua Xi Road, Department of Emergency Medicine and Center of Chest Pain, Qilu

Hospital of Shandong University, Jinan, 250012, P.R. China.

e-mail: warmsunlight2006@yahoo.com.cn 
the plaque/thrombus interface in erosions, compared to ruptures and stable plaques (Kolodgie et al. 2002), suggesting that these molecules exposed after endothelial denudation may be involved in acute thrombosis of eroded plaques. Therefore, we explored the potential mechanisms of endothelial denudation and then thrombosis in this study.

Theoretically, endothelial denudation can be caused by turbulent blood flow, vasospasm or increasing endothelial cell apoptosis (programmed cell death). Unfortunately, little experimental evidence is available to support these processes. An earlier study has demonstrated the elevated microparticles derived from apoptotic endothelial cells (ECs) in the circulating blood of the patients with ACS (Mallat et al. 2000). Additionally, high levels of circulating apoptotic ECs were present in patients with myocardial infarction and angina (Hladovec et al. 1978; Mutin et al. 1999). It has been also reported that endothelial apoptosis may directly affect blood thrombogenicity through the release of apoptotic microparticles into the bloodstream (Mallat and Tedgui 2001). Bombeli et al. (1997) found that apoptotic ECs became procoagulant by increasing expression of phosphatidylserine and the loss of anticoagulant membrane components. Another study (Bombeli et al. 1999) showed that apoptosis of human umbilical vein endothelial cells (HUVEC) was induced by staurosporine or by serum starvation, and that there was a marked increase in platelet accumulation and binding independent of the method of apoptosis induction.

All these studies mentioned above suggest a relationship between endothelial apoptosis and endothelial denudation and thrombosis (Hladovec et al. 1978; Bombeli et al. 1997; Bombeli et al. 1999; Mutin et al. 1999; Mallat and Tedgui 2001); however, direct evidence to link these events is still lacking. Thus, the aim of the present research is to provide this evidence using a rabbit model of atherosclerosis.

\section{Materials and Methods}

Animals

Thirty-seven male New Zealand White rabbits (weight $1.5 \sim 2$ $\mathrm{kg}$ ) underwent balloon-induced abdominal aorta wall injury and were then fed a diet of $1 \%$ cholesterol for 3 months to establish atherosclerotic plaques, as previously described (Chen et al. 2004). During the period, four died, in which two for excess anesthetic during the abdominal aorta operation, one for cerebral infarction and another for diarrhea. After 3 months, the survived 33 animals were randomized into three groups, and their plaque-rich abdominal aortae were randomly incubated with $5 \times 10^{-5} \mathrm{~mol} / \mathrm{L}$ (high concentration group, $n=$ 12 ) or $1 \times 10^{-5} \mathrm{~mol} / \mathrm{L}$ (low concentration group, $n=12$ ) staurosporine (Alexis, USA), or saline (control group, $n=9$ ). The study protocol was shown in Fig. 1. Animal welfare was approved by Qilu Hospital of Shandong University in compliance with the WHO International Guiding Principles for Animal Research.

Drug treatment of abdominal aorta

After three months of a high-cholesterol diet, we incised the abdomens of these rabbits and then examined abdominal aorta with
37 rabbits underwent post-balloon-injury three- month high-cholesterol feeding

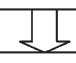

$2 \mathrm{~cm}$-length plaque-rich abdominal aortae were examined and then exposed to staurosporine or saline in vivo for $20 \mathrm{~min}$

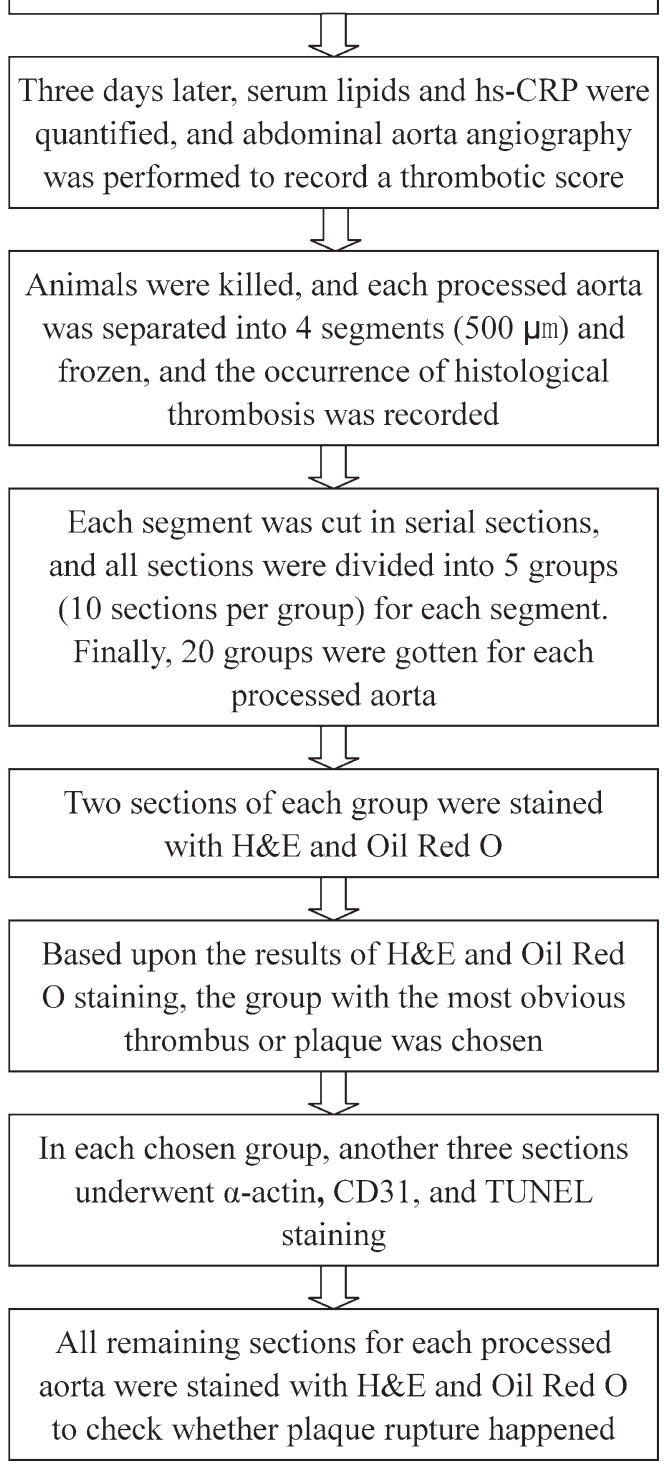

Fig. 1. Study protocol

high frequency vascular echocardiography, and chose a segment (2 $\mathrm{cm}$ long) with distinct plaques, and clamped it above and below the plaques with atraumatic vascular clips. Blood was withdrawn from the clamped segment with a $1 \mathrm{ml}$ needle, and approximately $300 \mu \mathrm{L}$ of staurosporine or saline was perfused with another needle. The region treated was marked with a suture nearby. After incubation for $20 \mathrm{~min}$, the segment was washed with saline 3 times, and then the clips were removed to expose this segment to the circulating blood for 3 days.

\section{Abdominal aorta angiography}

Three days after incubation, abdominal aorta angiography was 
performed blindly by two experienced interventional cardiologists to record a thrombotic score as followings: 0 , no thrombus; 1 , intraluminal haziness; 2, definite thrombus with its diameter or length $<0.5$ times normal vessel diameter; 3, 0.5-2 times; 4, > 2 times (Gurbel et al. 1996). Angiographic overt thrombosis was defined as having thrombotic scores of 3-4.

Measurement of serum lipid and high sensitive C-reactive protein $(h s-C R P)$

Abdominal aorta blood samples were taken by catheter during angiography. Serum levels of total cholesterol (TC), triglyceride (TG), low-density lipoprotein (LDL) and hs-CRP, which has been confirmed to be an inflammatory factor to accelerate coronary atherosclerosis (Ridker et al. 2005), were detected by enzymatic and immune turbidity assays, as previously described (Chen et al. 2006).

\section{Histopathology and immunohistochemistry}

The processed arteries were fixed with $4 \%$ paraformaldehyde for $24 \mathrm{~h}$, then were divided into 4 segments equally (500 $\mu \mathrm{m}$ per segment) and embedded in OCT medium. Frozen segments were cut in serial sections (10 $\mu \mathrm{m}$ thickness), and the occurrence of histological thrombosis was recorded during the procedure. For the arteries with angiographic thrombosis, only the thrombotic segments were cut continuously. For each segment, every 10 serial sections were set as a group, and so five groups could be obtained. Two sections of each group were stained with hematoxylin and eosin $(\mathrm{H} \& \mathrm{E})$ to evaluate the structure of the plaques and Oil Red O (Sigma Chemical Co., Saint Louis, USA) to show lipid deposition in the plaques. Based upon the results of H\&E and Oil Red O staining, the group with the most obvious thrombus was chosen in every segment with thrombi, and the group with the most obvious plaque was chosen in the one which had no thrombus. Another three sections in each chosen group underwent immunostaining and in situ detection of apoptotic cells with the followings: a mouse monoclonal anti-human $\alpha$-smooth muscle cell actin antibody (Boster Biological Technology Co. Ltd., Wuhan, China) to show smooth muscle cells, a polyclonal anti-CD31 antibody (Boster Biological Technology Co. Ltd., Wuhan, China) to show ECs, and TUNEL kits (Boster Biological Technology Co. Ltd., Wuhan, China) to show endothelial apoptosis. During the process of $\alpha$-smooth muscle cell actin and CD31 staining, a goat anti-mouse secondary antibody, horseradish peroxidase and diaminobenzidine were used after the development with first antibodies mentioned above, and then nuclear counterstaining was with hematoxylin. TUNEL staining was performed according to the protocol of the instruction of the kit. At last, all the remaining sections for every rabbit were stained with H\&E and Oil Red O.

Evaluation of endothelium integrity and eroded plaque

Endothelium integrity or endothelial denudation was evaluated according to the chosen section stained with CD31 in each aorta, and a quantitative imaging analysis package was used to analyze the percentages of remaining ECs.

All the sections stained with $\mathrm{H} \& \mathrm{E}$, Oil Red O, and $\alpha$-smooth muscle cell actin were detected to determine whether the lipid was connected with thrombi in the lumens, and whether the fibrous caps were complete and rich in smooth muscle cells.

\section{Endothelial apoptosis score}

A semiquantitative apoptosis score was estimated to evaluate the extent of endothelial cell apoptosis, as follows: 0 , no or barely detectable staining; 1 , weak positive staining; 2 , moderate limited staining; and 3, strong diffuse staining. The areas without ECs (negative CD31 staining) were considered as apoptosis-induced endothelial denudation, so they were also counted as apoptosis.

\section{Statistical analysis}

All quantitative data were expressed as mean $\pm \mathrm{SD}$, absolute numbers or percentages. Fisher's exact test, unpaired Student's $t$ test, one-way analysis of variance (ANOVA), Student Newman-Keuls (S-N-K) method and nonparametric Pearson correlation analysis were performed for corresponding data sets. Risk factors of thrombosis were evaluated using multiple logistic regression analysis. Differences were considered statistically significant at two-tailed $P<$ 0.05 .

\section{Results \\ Serum lipid and hs-CRP levels}

Table 1 shows the serum lipid and hs-CRP levels of abdominal aortas before angiography. There were no significant differences in terms of TC, TG, LDL-C and hs-CRP between the three groups.

\section{Angiographic thrombosis and histological thrombosis}

Abdominal aorta angiography revealed that there were higher thrombotic scores in rabbits treated with staurosporine relative to controls $(1.88 \pm 1.33$ in the rabbits treated with staurosporine, $2.33 \pm 1.37$ in the high concentration group, $1.42 \pm 1.16$ in the low concentration group vs. $0.11 \pm$ 0.33 in the control group; $P<0.01$, respectively); and the incidence of angiographic overt thrombosis was substantially higher in the rabbits treated with staurosporine $(9 / 24$, $37.5 \%)$ than that in the rabbits treated with saline $(0 / 9,0 \%)$ (Fig. 2). During the cutting of frozen segments, histological thrombosis was observed in $18(75.0 \%)$ of the rabbits treated with staurosporine, including $10(83.3 \%)$ in the high concentration group and $8(66.6 \%)$ in the low concentration group, but in only $1(11.1 \%)$ of the controls treated with

Table 1. Levels of serum lipids and hs-CRP before abdominal aorta angiography.

\begin{tabular}{lcccc}
\hline \multicolumn{1}{c}{ Group } & TC $(\mathrm{mmol} / \mathrm{L})$ & TG $(\mathrm{mmol} / \mathrm{L})$ & LDL-C $(\mathrm{mmol} / \mathrm{L})$ & $\mathrm{hs-CRP}(\mathrm{ng} / \mathrm{mL})$ \\
\hline High concentration group & $34.47 \pm 9.61$ & $1.81 \pm 1.04$ & $31.25 \pm 8.74$ & $139.1 \pm 46.6$ \\
Low concentration group & $31.82 \pm 8.44$ & $2.07 \pm 1.05$ & $27.02 \pm 7.29$ & $151.7 \pm 53.9$ \\
Controls & $31.92 \pm 8.47$ & $2.03 \pm 1.01$ & $28.25 \pm 6.53$ & $136.4 \pm 41.2$ \\
\hline
\end{tabular}
Protein.

TC, total cholesterol; TG, triglyceride; LDL, low-density lipoprotein; hs-CRP, High Sensitive C-reactive 

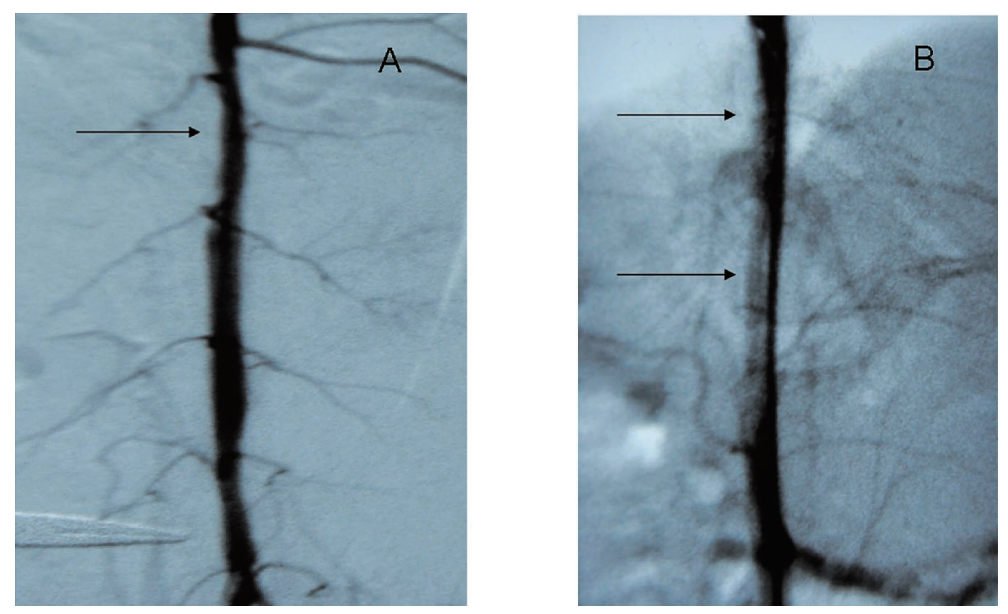

Fig. 2. Abdominal aorta angiography after 3 days of staurosporine or saline treatment.

A, An atherosclerotic stenosis in saline controls (arrow). B, An atherosclerotic stenosis with thrombi in staurosporinetriggered aortas (arrow).
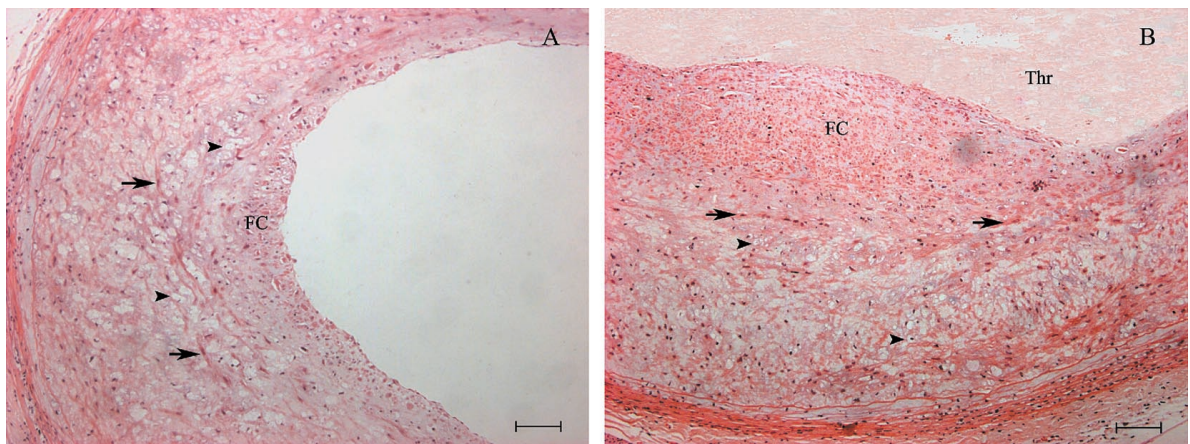

Fig. 3. Sections of atherosclerotic plaques with HE staining.

Endothelium is considerably complete in the control group (A) and yet incomplete in the staurosporine-triggered aortas with thrombi (B). There are massive spindle-shaped smooth muscle cells (arrows) and foam cells (arrowheads) in the plaques of A and B. FC: fibrous cap, Thr: thrombus. Bar $=100 \mu \mathrm{m}$.
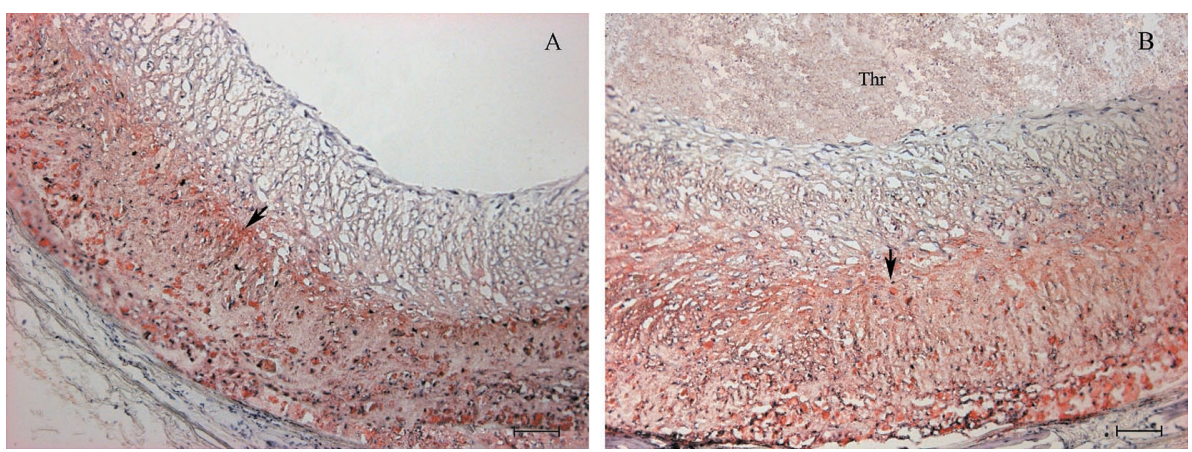

Fig. 4. Sections stained with Oil Red O.

A, A plaque with abundant lipid (arrow) in the control group. B, A plaque with lipid (arrow) and thrombus in the rabbits treated with staurosporine, but the lipid is in the deep of the plaque and not connected with luminal thrombus. Bar $=100$ $\mu \mathrm{m}$.

saline. As compared to the controls, all the rabbits treated with staurosporine and the high or low concentration groups had significantly higher occurrences of histological thrombosis $(P<0.01, P<0.01, P=0.02$, respectively).
Oil Red $O$ and $\alpha$-smooth muscle cell actin staining

H\&E staining revealed that every vessel had obvious plaques; all the plaques were rich in spindle-shaped smooth muscle cells and foam cells; no foam cells were in the lumens (Fig. 3). Oil Red O staining revealed that all the 

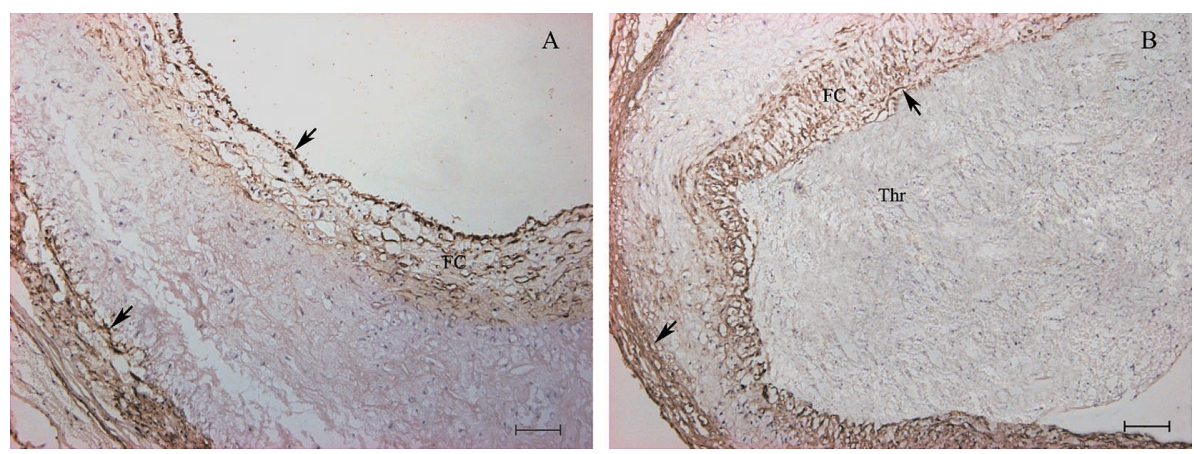

Fig. 5. Sections stained with $\alpha$-smooth muscle cell actin.

Positive staining (arrows) is confined to the media and the fibrous caps, and there are no ruptures in the smooth-musclecells-rich fibrous caps. A, The controls. B, The aortas treated with staurosporine. Bar $=100 \mu \mathrm{m}$.

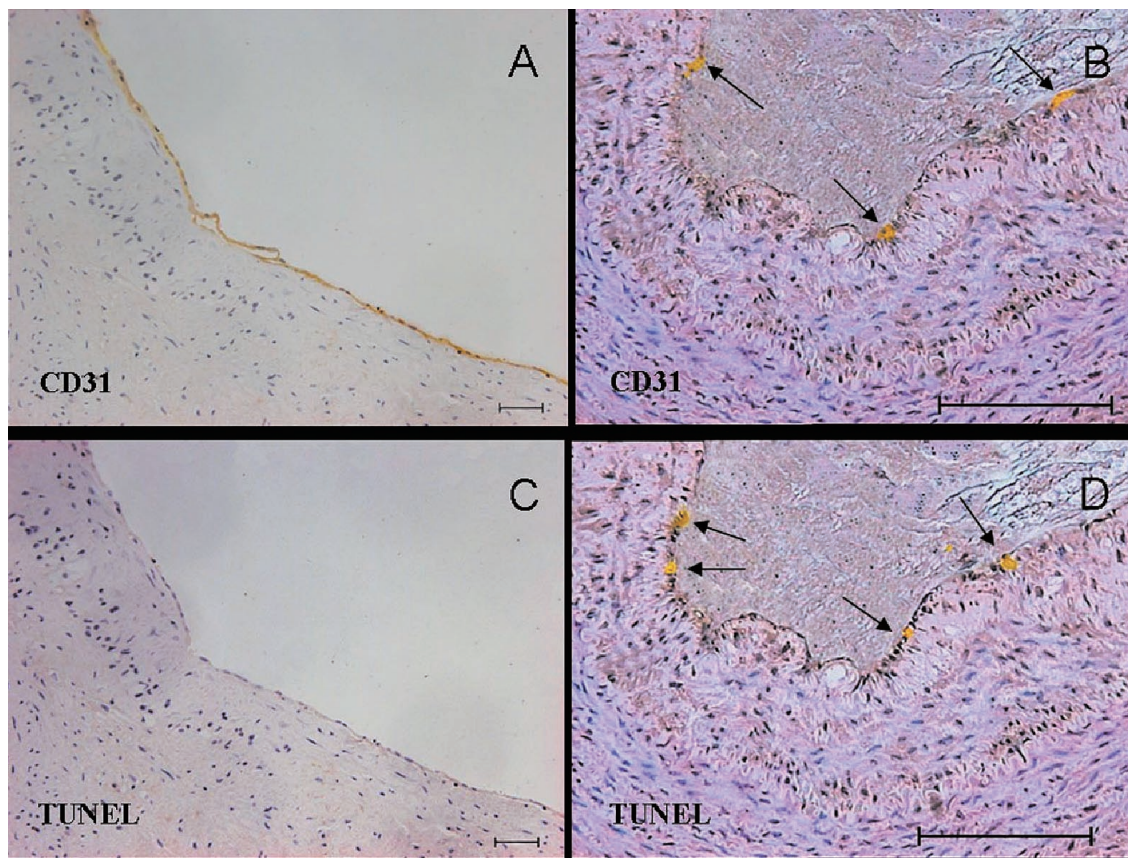

Fig. 6. Sections stained with CD31 and TUNEL.

In the control group, positive CD31 staining and negative TUNEL staining in adjacent sections of the same lesion are shown in panels $\mathrm{A}$ and $\mathrm{C}$ respectively; in the aortas treated with staurosporine, weak CD31 positive staining and distinct TUNEL positive staining at the plaque/thrombus interface are shown with arrows in panels B and D respectively. Bar = $100 \mu \mathrm{m}$.

plaques in each group contained abundant lipid under the fibrous caps, and the lipid was not associated with thrombi in the lumens for all the thrombogenic aortae (Fig. 4). $\alpha$ smooth muscle cell actin staining demonstrated that every specimen had apparent smooth muscle cell layers in media, and the fibrous caps richly contained smooth muscle cells (Fig. 5). H\&E, Oil Red $\mathrm{O}$ and $\alpha$-smooth muscle cell actin staining suggested that there was no rupture in fibrous caps and no lipid overflowed under thrombi in all the plaques treated with staurosporine.

\section{Endothelium integrity and apoptosis}

CD31 staining showed that there were considerably integrated endothelial monolayers at the surfaces of the plaques in the control group (Fig. 6A), but partial or large areas of endothelial monolayers denudation were observed in the two groups treated with staurosporine (Fig. 6B). TUNEL staining showed that apoptotic ECs were rare in the controls (Fig. 6C), while TUNEL positive staining (Fig. 6D) and areas of negative CD31 staining (Fig. 6B) were detectable frequently at the plaque/thrombus interface in rabbits treated with staurosporine.

The percentages of remaining ECs in the high and low concentration groups were both remarkably lower than that in the control group $(12.6 \pm 2.8$ in the rabbits treated with staurosporine, $10.1 \pm 2.5$ in the high concentration group and $14.2 \pm 2.4$ in the low concentration group vs. $90.7 \pm 4.1$ in controls; $P<0.01$, respectively). The rabbits treated with 


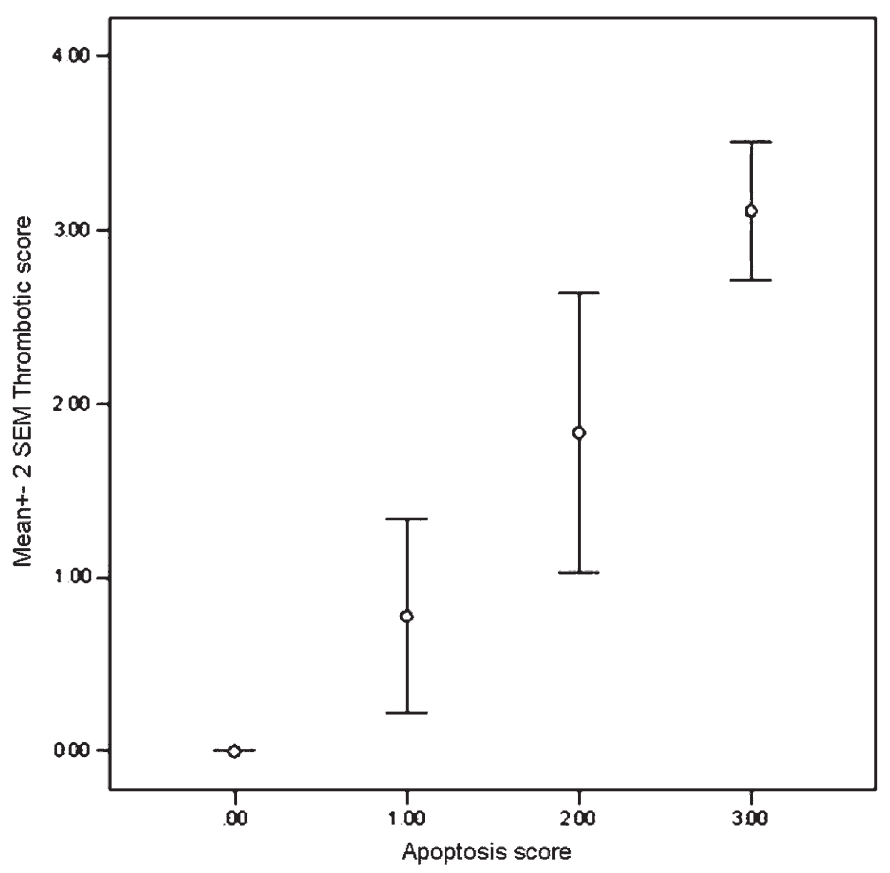

Fig. 7. Correlation between thrombosis and endothelial apoptosis.

The increased apoptosis score was linearly associated with the increased thrombotic score $(\mathrm{F}=103.49, P<0.0001)$.

staurosporine had significantly higher apoptosis scores compared to saline controls $(1.92 \pm 1.02$ in the rabbits treated with staurosporine, $2.42 \pm 0.79$ in the high concentration group, $1.42 \pm 1.00$ in the low concentration group vs. $0.22 \pm$ 0.44 in the control group; $P<0.01$, respectively).

\section{Correlation analyses of various factors and risk factors of thrombosis}

Using nonparametric Pearson correlation analysis, we showed significant association between apoptosis score and thrombotic score $(r=0.88, P<0.001)$. On the other hand, the endothelial integrity was reversely correlated with apoptosis score $(r=-0.91, P<0.001)$. Using One-way ANOVA model, we have further confirmed that the increased apoptosis score was linearly associated with the increased thrombotic score $(F=103.49, P<0.0001)$ (Fig. 7).

By including the variables of serum lipid and hs-CRP, staurosporine concentration, endothelial apoptosis and endothelial denudation in the multiple logistic regression analysis model, we found that endothelial apoptosis was an independent risk factor for histological thrombosis and angiographic overt thrombosis (OR $=13.75,95 \% \mathrm{CI}, 2.13$ 94.26, $P<0.01 ; \mathrm{OR}=40.25,95 \% \mathrm{CI}, 2.59 \sim 660.74, P<$ $0.01)$.

\section{Discussion}

Culprit coronary atherosclerotic lesions, which are responsible for ischemic coronary disease, are usually classified into ruptured plaque, eroded plaque, or calcific nodule (Naghavi et al. 2003a; Naghavi et al. 2003b). A study of morphological classification of atherosclerotic lesions showed that plaques histologically resembling erosion were found proximal to the ruptured site in some culprit lesions, which indicates that the identification of eroded plaques can be complicated and confusing (Virmani et al. 2000). Aside from the absence of rupture in the fibrous cap, other main characteristics of eroded plaque are as follows: (1) endothelial denudation at the plaque/thrombus interface; (2) deep lipid pools which don't communicate with the luminal thrombus; and (3) an abundance of smooth muscle cells and proteoglycans in plaques (Virmani et al. 2000; Naghavi et al. 2003a; Naghavi et al. 2003b). Thus, a careful assessment of the plaque with the characteristics mentioned above should be first performed before any further investigations about eroded plaque. In our study, we have identified whether the rupture of caps and lipid overflow happened by serial sections and staining, and then we have shown that endothelial apoptosis is indeed involved in the formation of thrombotic eroded atherosclerotic plaques.

Staurosporine, an inhibitor of protein kinase C, blocks cell metabolism, thereby inducing apoptosis. EC apoptosis triggered by staurosporine incubation or serum starvation showed similar outcomes in relation to the severity and pattern of apoptosis (Bombeli et al. 1999). Accordingly, staurosporine was chosen as the drug for establishing the eroded plaque model in our research. In this study, we detected characteristics of staurosporine-induced plaques with serial sections and Oil Red $\mathrm{O}, \alpha$-smooth muscle cell actin and CD31 staining. No fibrous cap rupture and lipid overflow into lumens were detected in all sections, and obvious endothelial denudation at the plaque/thrombus interface was observed frequently in these sections. Additionally, there 
was abundant positive $\alpha$-smooth muscle cell actin staining in these thick non-calcified fibrous caps. These characteristics are all similar to those of human eroded plaques. As far as we are aware, this is one of a few eroded plaque models available published so far (Naghavi et al. 2003a; Naghavi et al. 2003b; Hayashi et al. 2005).

Endothelial denudation is a critical feature of eroded plaques. Endothelial injury and recovery occurs throughout the initiation and maintenance of atherosclerotic vessel development. Nevertheless, there are no large areas of spontaneous endothelial denudation, even in advanced human atherosclerotic plaques (Davies et al. 1988). Therefore, endothelial cell denudation at the interface of eroded-plaque/thrombus is a pathologic, not physiologic event, and the investigation about this must contribute to the realization of eroded plaque. Some studies suggest there is increasing endothelial apoptosis in the patients with ischemic coronary disease (Hladovec et al. 1978; Mutin et al. 1999; Mallat et al. 2000). Furthermore, previous studies have suggested that endothelial apoptosis could contribute directly to thrombogenesis (Bombeli et al. 1997; Bombeli et al. 1999; Mallat and Tedgui 2001). However, up to now, direct evidence in appropriate eroded atherosclerotic plaque model has not been shown for the relationship between endothelial apoptosis and significant endothelial denudation or thrombogenesis.

In our study, we found that there were less remaining ECs, higher thrombotic scores, more histological thrombosis and angiographic overt thrombosis in the erosion-similar plaques treated with staurosporine relative to atherosclerotic controls treated with saline. And the endothelial apoptosis score in the staurosporine-induced plaques was also higher than that in the controls. These findings indicate that apoptotic ECs may induce endothelial denudation and thrombosis in eroded plaques.

An early study with a small sample showed that half of the eroded plaque samples contained significant inflammatory infiltration, while the other half contained mild infiltration (van der Wal et al. 1994). However, in other larger sample studies, mild inflammatory cell infiltration in eroded plaques was observed (Arbustini et al. 1999; Virmani et al. 2000; Virmani et al. 2001). Additionally, the lipid hypothesis has also been accepted widely with respect to the occurrence and development of atherosclerosis (Rosenson and Lowe 1998). Nevertheless, in our study, there were no significant differences in serum hs-CRP, TC, TG and LDL-C between different groups, while there was a significant correlation between EC apoptosis and thrombosis, which highlights the independent role of apoptotic ECs in eroded plaques with thrombosis.

Our study further showed that there was a reverse relationship between endothelial apoptosis and endothelium integrity and a positive relation between endothelial apoptosis and thrombosis, and the increased apoptosis score was linearly associated with the increased thrombotic score. Furthermore, logistic multiple analysis demonstrated that endothelial cell apoptosis was an independent risk factor for histological thrombosis and angiographic overt thrombosis in eroded plaques, which excluded the potential effects of staurosporine toxicity, lipids, inflammation and plaque matrix exposed after endothelial denudation. Therefore, all these results in our study strengthen the significance of endothelial cell apoptosis in thrombotic eroded plaque. Further studies are needed to establish the causal relationship between endothelial apoptosis and thrombotic eroded plaque.

Although our study suggests that endothelial cell apoptosis could induce thrombotic eroded plaque, factors that aggravate and accelerate apoptosis in patients with thrombotic eroded plaque are still unknown. Some studies suggest that patients who die of eroded plaque are often smokers (Virmani et al. 1999). Recently, a study showed that polycyclic aromatic hydrocarbons found in cigarettes could induce human coronary artery endothelial cell apoptosis (Tithof et al. 2002). In addition, despite the lack of evidence that turbulent blood flow directly induces endothelial denudation, an in vivo study suggested that there was a systemic preferential occurrence of apoptosis in the downstream parts of plaques, where low flow and low shear stress prevail (Tricot et al. 2000). Unfortunately, the predilection site of eroded plaques in humans has not been well elucidated until now. It has been also reported that hypoxia, oxLDL, homocysteine, oxygen-derived free radicals and some cytokines in blood can induce endothelial cell apoptosis (Isner et al. 1995; Konno et al. 1999; Mercie et al. 2000; Lee et al. 2005). Some researchers also demonstrated that apoptosis-induced genes like Bax, Fas and p53 were upregulated in atherosclerotic plaques (Kockx 1998). All factors mentioned above might play a role in endothelial denudation and thrombosis of eroded plaque by promoting endothelial cell apoptosis. However, further studies are needed to elucidate specific factors essential to thrombotic eroded plaque so that more strategies could be found to prevent sudden coronary death.

In conclusion, we established a rabbit model of eroded plaques using the staurosporine-induction approach and investigated the underlying role of endothelial apoptosis in eroded plaques. Our study suggests that endothelial apoptosis contributes to the formation of thrombotic eroded plaque. All the work would be beneficial to the understanding and further researches about culprit lesions without rupture of patients with sudden coronary death or other acute coronary events.

\section{Acknowledgments}

This study was supported by a Key Project from Department of Science and Technology of Shandong Province, China (2005GG4402047). The operations on animals were made with the help of Dr. Xi-sheng Xu, Dr. Xin-yan Pang, Dr. Guo-yong Zhang and Dr. Hai-feng Zhang. 


\section{References}

Arbustini, E., Dal Bello, B., Morbini, P., Burke, A.P., Bocciarelli, M., Specchia, G. \& Virmani, R. (1999) Plaque erosion is a major substrate for coronary thrombosis in acute myocardial infarction. Heart, 82, 269-272.

Bombeli, T., Karsan, A., Tait, J.F. \& Harlan, J.M. (1997) Apoptotic vascular endothelial cells become procoagulant. Blood, $\mathbf{8 9}$, 2429-2442.

Bombeli, T., Schwartz, B.R. \& Harlan, J.M. (1999) Endothelial cells undergoing apoptosis become proadhesive for nonactivated platelets. Blood, 93, 3831-3838.

Chen, W.Q., Zhang, Y., Zhang, M., Ji, X.P., Yin, Y. \& Zhu, Y.F. (2004) Establishing an animal model of unstable atherosclerotic plaques. Chin. Med.J (Engl)., 117, 1293-1298.

Chen, Y.G., Xu, F., Zhang, Y., Ji, Q.S., Sun, Y., Lu, R.J. \& Li, R.J. (2006) Effect of aspirin plus clopidogrel on inflammatory markers in patients with non-ST-segment elevation acute coronary syndrome. Chin. Med. J (Engl)., 119, 32-36.

Davies, M.J., Woolf, N., Rowles, P.M. \& Pepper, J. (1988) Morphology of the endothelium over atherosclerotic plaques in human coronary arteries. Br. Heart J., 60, 459-464.

Farb, A., Burke, A.P., Tang, A.L., Liang, T.Y., Mannan, P., Smialek, J. \& Virmani, R. (1996) Coronary plaque erosion without rupture into a lipid core. A frequent cause of coronary thrombosis in sudden coronary death. Circulation, 93, 1354-1363.

Gurbel, P.A., Navetta, F.I., Bates, E.R., Muller, D.W., Tenaglia, A.N., Miller, M.J., Muhlstein, B., Hermiller, J.B., Davidson, C.J., Aguirre, F.V., Beauman, G.J., Berdan, L.G., Leimberger, J.D., Bovill, E.G., Christenson, R.H. \& Ohman, E.M. (1996) Lesion-directed administration of alteplase with intracoronary heparin in patients with unstable angina and coronary thrombus undergoing angioplasty. Cathet. Cardiovasc. Diagn., 37, 382-391.

Hayashi, T., Kiyoshima, T., Matsuura, M., Ueno, M., Kobayashi, N., Yabushita, H., Kurooka, A., Taniguchi, M., Miyataka, M., Kimura, A. \& Ishikawa, K. (2005) Plaque erosion in the culprit lesion is prone to develop a smaller myocardial infarction size compared with plaque rupture. Am. Heart J., 149, 284290.

Hladovec, J., Prerovsky, I., Stanek, V. \& Fabian, J. (1978) Circulating endothelial cells in acute myocardial infarction and angina pectoris. Klin. Wochenschr., 56, 1033-1036.

Isner, J.M., Kearney, M., Bortman, S. \& Passeri, J. (1995) Apoptosis in human atherosclerosis and restenosis. Circulation, 91 , 2703-2711.

Kockx, M.M. (1998) Apoptosis in the atherosclerotic plaque: quantitative and qualitative aspects. Arterioscler. Thromb. Vasc. Biol., 18, 1519-1522.

Kolodgie, F.D., Burke, A.P., Farb, A., Weber, D.K., Kutys, R., Wight, T.N. \& Virmani, R. (2002) Differential accumulation of proteoglycans and hyaluronan in culprit lesions: insights into plaque erosion. Arterioscler. Thromb. Vasc. Biol., 22, 1642-1648.

Kolodgie, F.D., Burke, A.P., Wight, T.N. \& Virmani, R. (2004) The accumulation of specific types of proteoglycans in eroded plaques: a role in coronary thrombosis in the absence of rupture. Curr. Opin. Lipidol., 15, 575-582.

Konno, R., Igarashi, T., Okamoto, S., Sato, S., Moriya, T., Sasano, H. \& Yajima, A. (1999) Apoptosis of human endometrium mediated by perforin and granzyme B of NK cells and cytotoxic T lymphocytes. Tohoku J. Exp. Med., 187, 149-155.

Lee, C.N., Cheng, W.F., Chang, M.C., Su, Y.N., Chen, C.A. \& Hsieh, F.J. (2005) Hypoxia-induced apoptosis in endothelial cells and embryonic stem cells. Apoptosis, 10, 887-894.

Mallat, Z., Benamer, H., Hugel, B., Benessiano, J., Steg, P.G., Freyssinet, J.M. \& Tedgui, A. (2000) Elevated levels of shed membrane microparticles with procoagulant potential in the peripheral circulating blood of patients with acute coronary syndromes. Circulation, 101, 841-843.

Mallat, Z. \& Tedgui, A. (2001) Current perspective on the role of apoptosis in atherothrombotic disease. Circ. Res., 88, 9981003

Mercie, P., Garnier, O., Lascoste, L., Renard, M., Closse, C., Durrieu, F., Marit, G., Boisseau, R.M. \& Belloc, F. (2000) Homocysteine-thiolactone induces caspase-independent vascular endothelial cell death with apoptotic features. Apoptosis, $\mathbf{5}$, 403-411.

Mutin, M., Canavy, I., Blann, A., Bory, M., Sampol, J. \& Dignat-George, F. (1999) Direct evidence of endothelial injury in acute myocardial infarction and unstable angina by demonstration of circulating endothelial cells. Blood, 93, 2951-2958.

Naghavi, M., Libby, P., Falk, E., Casscells, S.W., Litovsky, S., Rumberger, J., Badimon, J.J., Stefanadis, C., Moreno, P., Pasterkamp, G., Fayad, Z., Stone, P.H., Waxman, S., Raggi, P., Madjid, M., Zarrabi, A., Burke, A., Yuan, C., Fitzgerald, P.J., Siscovick, D.S., de Korte, C.L., Aikawa, M., Airaksinen, K.E., Assmann, G., Becker, C.R., Chesebro, J.H., Farb, A., Galis, Z.S., Jackson, C., Jang, I.K., Koenig, W., Lodder, R.A., March, K., Demirovic, J., Navab, M., Priori, S.G., Rekhter, M.D., Bahr, R., Grundy, S.M., Mehran, R., Colombo, A., Boerwinkle, E., Ballantyne, C., Insull, W., Jr., Schwartz, R.S., Vogel, R., Serruys, P.W., Hansson, G.K., Faxon, D.P., Kaul, S., Drexler, H., Greenland, P., Muller, J.E., Virmani, R., Ridker, P.M., Zipes, D.P., Shah, P.K. \& Willerson, J.T. (2003a) From vulnerable plaque to vulnerable patient: a call for new definitions and risk assessment strategies: Part II. Circulation, 108, 1772-1778

Naghavi, M., Libby, P., Falk, E., Casscells, S.W., Litovsky, S., Rumberger, J., Badimon, J.J., Stefanadis, C., Moreno, P., Pasterkamp, G., Fayad, Z., Stone, P.H., Waxman, S., Raggi, P., Madjid, M., Zarrabi, A., Burke, A., Yuan, C., Fitzgerald, P.J., Siscovick, D.S., de Korte, C.L., Aikawa, M., Juhani Airaksinen, K.E., Assmann, G., Becker, C.R., Chesebro, J.H., Farb, A., Galis, Z.S., Jackson, C., Jang, I.K., Koenig, W., Lodder, R.A., March, K., Demirovic, J., Navab, M., Priori, S.G., Rekhter, M.D., Bahr, R., Grundy, S.M., Mehran, R., Colombo, A., Boerwinkle, E., Ballantyne, C., Insull, W., Jr., Schwartz, R.S., Vogel, R., Serruys, P.W., Hansson, G.K., Faxon, D.P., Kaul, S., Drexler, H., Greenland, P., Muller, J.E., Virmani, R., Ridker, P.M., Zipes, D.P., Shah, P.K. \& Willerson, J.T. (2003b) From vulnerable plaque to vulnerable patient: a call for new definitions and risk assessment strategies: Part I. Circulation, 108, 1664-1672.

Ridker, P.M., Cannon, C.P., Morrow, D., Rifai, N., Rose, L.M., McCabe, C.H., Pfeffer, M.A. \& Braunwald, E. (2005) C-reactive protein levels and outcomes after statin therapy. N. Engl. J. Med., 352, 20-28.

Rosenson, R.S. \& Lowe, G.D. (1998) Effects of lipids and lipoproteins on thrombosis and rheology. Atherosclerosis, 140, 271280.

Tithof, P.K., Elgayyar, M., Cho, Y., Guan, W., Fisher, A.B. \& Peters-Golden, M. (2002) Polycyclic aromatic hydrocarbons present in cigarette smoke cause endothelial cell apoptosis by a phospholipase A2-dependent mechanism. Faseb. J., 16, 1463-1464.

Tricot, O., Mallat, Z., Heymes, C., Belmin, J., Leseche, G. \& Tedgui, A. (2000) Relation between endothelial cell apoptosis and blood flow direction in human atherosclerotic plaques. Circulation, 101, 2450-2453.

van der Wal, A.C., Becker, A.E., van der Loos, C.M. \& Das, P.K. (1994) Site of intimal rupture or erosion of thrombosed coronary atherosclerotic plaques is characterized by an inflammatory process irrespective of the dominant plaque morphology. Circulation, 89, 36-44.

Virmani, R., Burke, A.P. \& Farb, A. (1999) Plaque rupture and plaque erosion. Thromb. Haemost., 82 Suppl 1, 1-3.

Virmani, R., Kolodgie, F.D., Burke, A.P., Farb, A. \& Schwartz, S.M. 
(2000) Lessons from sudden coronary death: a comprehensive morphological classification scheme for atherosclerotic lesions. Arterioscler. Thromb. Vasc. Biol., 20, 1262-1275.
Virmani, R., Burke, A.P. \& Farb, A. (2001) Sudden cardiac death. Cardiovasc. Pathol., 10, 211-218. 